\title{
Racial Disparities in the Quality of Medication Use in Older Adults: Baseline Findings from a Longitudinal Study
}

\author{
Mary T. Roth, PharmD, $M H S^{7}$, Denise A. Esserman, $P h D^{2}$, Jena L. Ivey, PharmD ${ }^{3}$, and Morris \\ Weinberger, $P h D^{4}$
}

'Division of Pharmaceutical Outcomes and Policy, UNC Eshelman School of Pharmacy, University of North Carolina at Chapel Hill, Chapel Hill, NC, USA; ${ }^{2}$ Division of General Medicine and Clinical Epidemiology, School of Medicine and Department of Biostatistics, UNC Gillings School of Global Public Health, University of North Carolina at Chapel Hill, Chapel Hill, NC, USA; ${ }^{3}$ Division of Pharmacy Practice and Experiential Education, UNC Eshelman School of Pharmacy, University of North Carolina at Chapel Hill, Chapel Hill, NC, USA; ${ }^{4}$ Department of Health Policy and Management, UNC Gillings School of Global Public Health, University of North Carolina at Chapel Hill, Chapel Hill, North Carolina and Senior Career Scientist, Durham VAMC Center for Health Services Research, Durham, NC, USA.

BACKGROUND: Medication-related problems are prevalent in older adults and adversely affect the quality of care. It has been suggested that racial differences exist in medication use. Most efforts to evaluate the quality of medication use target specific drugs or disease states, or a set of pre-defined quality indicators, rather than the patient.

OBJECTIVE: We conducted a prospective cohort study to determine the prevalence and types of medicationrelated problems in older adults, examining the impact of race on quality medication use.

METHODS: In-home interviews and medical record reviews of 200 (100 white, 100 black) older adults were conducted three times over 1 year. The quality of medication use was measured using a clinical pharmacist's assessment of quality and the Assessing Care of Vulnerable Elders quality indicators. We used logistic and negative binomial regression models to analyze the two primary endpoints of prevalence and number of medication-related problems.

RESULTS: Mean age was 78.3 (whites) and 75.5 (blacks), with the majority being female. Although whites used more medications than blacks ( 11.6 versus 9.7; $\mathrm{p}<0.01$ ), blacks had more medication-related problems per person than whites $(6.2$ versus 4.9 ; $\mathrm{p}<0.01)$. All patients had at least one medication-related problem; undertreatment, suboptimal drug, suboptimal dosing, and nonadherence were most prevalent. Blacks had significantly higher rates of nonadherence than whites (68\% versus $42 \%$; $\mathrm{p}<0.01)$.

CONCLUSION: Medication-related problems are prevalent in community-residing older adults. Blacks had more medication-related problems than whites, including higher rates of nonadherence. These findings require further study to better understand racial disparities in quality medication use.

Received May 26, 2009

Revised October 13, 2009

Accepted October 20, 2009

Published online December 11, 2009
KEY WORDS: quality; medications; race; disparities; elderly.

$\mathrm{J}$ Gen Intern Med 25(3):228-34

DOI: $10.1007 / \mathrm{s} 11606-009-1180-9$

(c) Society of General Internal Medicine 2009

\section{BACKGROUND}

Older adults are more likely to have multiple co-existing chronic diseases, visit more than one prescriber, and use multiple medications, placing them at an increased risk of developing medication-related problems. This, in turn, may compromise their health status, functional status, and quality of life. ${ }^{1-3}$ Moreover, the costs associated with drug-related morbidity and mortality are staggering, estimated in one study to be $\$ 177$ billion annually, with nearly half ( $\$ 80$ billion) in ambulatory care. ${ }^{4-7}$ The effect of race on the quality of medication use is unknown, although it has been suggested that racial differences exist in medication use. ${ }^{8}$ For example, older black adults have been found to have lower total drug spending, use fewer prescription medications, and have higher rates of nonadherence than whites. ${ }^{9,10}$

Traditional efforts to measure the quality of medication use often focus on select medication-related problems or predefined quality indicators; however, such an approach fails to consider the multiple, co-existing problems within individuals. ${ }^{11,12}$ A more comprehensive, individualized approach to evaluating and managing medications may be more effective in improving the overall quality of medication use. Such an approach is especially needed for older adults who have complex medication regimens and are at increased risk for experiencing medication-related problems.

Adapted from the Institute of Medicine's definition of quality of care, the quality of medication use can be defined as "the degree to which medication use for individuals and populations increases the likelihood of desired health outcomes and is consistent with current professional knowledge." ${ }^{13,14} \mathrm{We}$ present baseline data from a longitudinal study of communitydwelling older adults, illustrating racial differences in the quality of medication use overall and by type of problem. 


\section{METHODS}

\section{Overview}

The detailed methods for the longitudinal study are described elsewhere. ${ }^{15}$ Patients were recruited from the Department on Aging Eldercare Program and two senior housing complexes located in Orange County, North Carolina. Subjects met the following inclusion criteria: (1) age $\geq 60$ years, (2) residing independently in the community setting, (3) taking $\geq 3$ regularly scheduled prescription and/or non-prescription medications, (4) able to read and speak English, and (5) willing to participate, as indicated by providing informed consent and HIPAA-compliant authorization for release of medical information. Patients were excluded if they had $\geq 3$ errors on a cognitive screening instrument ${ }^{16}$ or had received clinical pharmacy services within the past 6 months. Eligible individuals (100 white and 100 black) were interviewed in their homes at baseline, 6 months, and 12 months. The study was conducted from April 2005 through August 2007. The Biomedical Institutional Review Board of the University of North Carolina at Chapel Hill approved this study.

The study employed two part-time pharmacists with Doctor of Pharmacy degrees. One completed residency training in geriatrics, is a board-certified pharmacotherapy specialist, and has practiced as a clinical pharmacist in an outpatient, interdisciplinary geriatric practice setting for 5 years; the other is an independent consultant pharmacist providing medication management services to older adults in the community.

\section{Participant Recruitment}

Using the Eldercare Program contact list, a screening call was conducted with each older adult to describe the study and invite them to participate in a baseline visit for verification of eligibility and enrollment. Interested individuals from the two senior housing complexes responded by telephone to posted flyers. The pharmacists conducted screening telephone calls, and, for individuals interested in participating, arranged a home visit to discuss the study, verify eligibility, obtain informed consent and HIPAA-compliant authorization for release of medical information, and enroll eligible individuals into the study. Of 435 older adults screened via telephone, 235 individuals were excluded because they were not eligible (105), could not be reached by phone (72), refused to participate (44), or had died (14). Thus, 200 patients were enrolled into the study and interviewed by a pharmacist.

\section{Data Collection and Measures}

All measures used in this study have been described in detail elsewhere. ${ }^{15}$ They include demographics; the short test of functional health literacy in adults (S-TOFHLA), which categorizes patient's ability to read and understand health-related material as inadequate (0-16), marginal (17-22), or adequate (23-36) health literacy; ${ }^{17}$ and an eight-item self-reported instrumental activities of daily living (IADL) scale that ranged from 0 (poorest) to 16 (best) functional status. ${ }^{18}$ Each item was scored as 0 (completely dependent on others), 1 (some assistance required), or 2 (independence), with items summed (maximum $=16$ ) to compute an IADL total score.

The quality of medication use was measured in two ways. First, the pharmacist used a three-step implicit process: (1) a comprehensive medication review with the older adult, (2) a medical record review, and (3) formulation of an assessment of quality medication use. During the comprehensive medication review, the pharmacist recorded detailed information on medical conditions, medications, and medication-taking behaviors. ${ }^{15}$ Following the home visit, the pharmacist conducted a medical record review to abstract information on medications, medical conditions, laboratory values, physician assessment of the individual's medical conditions, hospitalizations, and any other information pertinent to assessing the individual's quality of medication use. For the final step, using data from the comprehensive medication review and medical record review, the pharmacist formulated an assessment of quality medication use by integrating best research evidence (e.g., published literature, established indicators of quality care, clinical practice guidelines) with clinical expertise and patient values. This assessment was guided by a list of potential medication-related problems (Table 1) that was

Table 1. Framework for Assessing Medication-Related Problems as a Measure of the Quality of Medication Use in Community-residing Older Adults

\begin{tabular}{l}
\hline Potential medication-related \\
problem \\
\hline Suboptimal drug \\
\\
Suboptimal dose, duration, \\
frequency, or \\
administration
\end{tabular}

Adverse drug events

Nonadherence

Definition

The individual is receiving a drug that has no indication, is not effective, or is potentially not safe (i.e., risk of using drug outweighs benefit)

The individual is taking an appropriate medication, but the dose, duration, frequency, or administration is not optimal to achieve the desired response, or has the potential for harm

The individual is experiencing adverse consequences attributed to a drug or the inappropriate use of a drug

The individual has not filled a prescription, is not taking a drug, or is not using a drug as prescribed, whether intentional or unintentional

Less costly drug available

The individual is prescribed a medication for which a less costly, equally effective and safe drug is available, and preferred by the patient, but the patient is receiving a more expensive product; or the patient could benefit from prescription assistance, but is not receiving the benefit and desires to

Undertreatment

The individual has a medical condition or risk factors for a disease that would benefit from drug therapy (clear indication) and the patient has no contraindications to the drug, but the drug was not prescribed

Suboptimal medication monitoring

The individual is receiving a drug and monitoring is recommended (according to established practice guidelines or quality indicators) to assess response to therapy or prevent harm, but has not been done

Adapted from Am J Geriatr Pharmacother.2008;6:220-233 
developed by study investigators following an extensive literature review. The categories in Table 1 were adapted from a previously published model to incorporate current professional knowledge and clinical experience. ${ }^{19-21}$

Second, to complement the implicit assessment, the pharmacist completed an explicit assessment during the medical record review using the Assessing Care of Vulnerable Elders (ACOVE-2) quality indicators. ${ }^{12}$ ACOVE-2 consists of 39 quality indicators of medication use categorized into four domains: (1) prescribing indicated medications, (2) avoiding inappropriate medications, (3) education, continuity, and documentation, and (4) medication monitoring. The ACOVE-2 indicators have excellent face validity due to the expert consensus process used in their development.

Adherence was assessed in two ways: (1) pharmacist assessment of adherence and (2) a validated patient self-report measure. ${ }^{22}$ The pharmacist's assessment was determined during the medication review for each chronic, maintenance medication a person was taking and based on both patient self-report and inspection of pill bottles. For each chronic medication, the pharmacist recorded "adherent" or "nonadherent." Any assessment of nonadherence was then documented as a medication-related problem in the category of nonadherence. Patient self-report was based on a validated four-item measure of medication-taking behavior over the past 4 weeks. $^{22}$

\section{Statistical Analysis}

The sample size estimation for our study was based on confidence interval (CI) widths and detecting meaningful differences between whites and blacks in the proportion of individuals with $\geq 1$ medication-related problem at baseline. A sample size of 100 per group met the conservative "sufficiently large" criterion formula. ${ }^{23}$

Descriptive statistics are presented, and comparisons were made by race (we oversampled blacks to specifically address racial differences) using two sample t-tests (continuous data) or chi-square tests of association (categorical data). The two primary outcomes were: (1) overall prevalence of medicationrelated problems in the population (proportion of subjects with any of the medication-related problems) and (2) average number of medication-related problems per person. Secondary outcomes were the prevalence and average number of problems broken down by the seven individual medicationrelated problems. The assessment of quality medication use using the ACOVE-2 quality indicators was included, but was not the primary outcome of this study; findings based on the ACOVE-2 quality indicators are beyond the scope of this paper, but will be the focus of subsequent work.

Logistic regression models were used to model the prevalence of each of the individual medication-related problems. Negative binomial models were used for the outcome of total number of medication-related problems (and each individual medicationrelated problem) since a Poisson regression did not provide a good fit to the data (large overdispersion). The primary "exposure" of interest was race. Due to pharmacist variability, models were adjusted for pharmacist and the following covariates identified $a$ priori based on published literature suggesting that these variables may have an impact on one or more medicationrelated problems: number of medications currently being taken by the individual, S-TOFHLA, age, and gender. For the primary outcomes of interest, statistical significance was achieved at a level of $\mathrm{p} \leq 0.05$. No formal corrections for multiple testing were conducted for the secondary outcomes of interest; however, we were conservative. Tests were considered statistically significant at a level of 0.01 , whereas tests with $0.01<$ p-value $\leq 0.05$ were considered suggestive of a relationship, but were not given as much weight. All analyses were conducted using SAS version 9.1 (SAS, Cary, NC).

\section{RESULTS}

Baseline demographic characteristics for the 200 subjects are presented in Table 2 . Whites were significantly older, had more education, and were more likely to live alone. Whites used more medications (11.6 versus 9.7, $\mathrm{p}<0.01$ ), had a greater number of chronic conditions (8.4 versus $7.4, \mathrm{p}=0.01)$, and used more physicians (3.6 versus $2.8,<0.01$ ) compared to blacks (Table 3). In addition, more whites maintained a written list of their medications than blacks (30\% versus $16 \%, \mathrm{p}=$ 0.02), whereas a higher percentage of blacks could not purchase their medications because of cost $(28 \%$ versus $12 \%$, $\mathrm{p}<0.01)$. Whites were more likely than blacks to have adequate health literacy ( $58 \%$ vs. $29 \%, \mathrm{p}<0.01)$.

\section{Quality of Medication Use}

The overall prevalence of problems was $100 \%$, that is, each participant had at least one medication-related problem documented at baseline. The most common problems cited for whites and blacks (Fig. 1) were undertreatment $(83 \%$ versus $87 \%$ ), suboptimal drug use (59\% versus $66 \%$ ), suboptimal dosing (48\% versus 56\%), and nonadherence $(42 \%$ versus $68 \%$ ). A significant difference in the prevalence of medication-related problems between whites and blacks was noted for nonadherence $(\mathrm{p}<0.01)$, with a smaller difference noted for suboptimal medication monitoring (15\% whites, 29\% blacks; $\mathrm{p}=0.05$ ). These findings were unaltered by adjustment for a priori potential confounders.

Although blacks were prescribed significantly fewer medications than whites, they had significantly more problems than whites (6.2 versus $4.9, \mathrm{p}<0.01$ ) (Table 4). A large part, but not all, of this difference can be attributed to the difference in nonadherence $(0.90$ whites, 1.9 blacks; $\mathrm{p}<0.01)$. These differences

Table 2. Demographic and Baseline Characteristics of Community-Residing Older Adults $(n=200)$

\begin{tabular}{llll}
\hline \hline & $\begin{array}{l}\text { White } \\
(\mathrm{n}=100)\end{array}$ & $\begin{array}{l}\text { Black } \\
(\mathrm{n}=100)\end{array}$ & P-value \\
\hline Age, mean (SD), years & $78.3(8.2)$ & $75.5(8.5)$ & 0.02 \\
Female, \% & 72 & 81 & 0.13 \\
Education, highest level & & & $<0.001$ \\
$\quad$ completed, \% & 8 & 24 & \\
Elementary & 6 & 19 & \\
Some high school & 21 & 35 & \\
High school graduate & 24 & 12 & \\
Some college or technical & & & \\
$\quad$ school & 18 & 6 & 0.03 \\
College graduate & 23 & 4 & \\
Postgraduate & 64 & 49 & \\
Living alone, \% & & &
\end{tabular}


Table 3. Medication and Health-Related Characteristics of Community-Residing Older Adults $(n=200)$

\begin{tabular}{|c|c|c|c|}
\hline & White $(n=100)$ & Black $(n=100)$ & P-value \\
\hline Medications, ${ }^{\mathrm{a}}$ mean (SD); range & 11.6 (5.0); 3-26 & $9.7(4) ; 4-21$ & 0.003 \\
\hline Chronic conditions, ${ }^{\mathrm{b}}$ mean (SD); range & 8.4 (3.1); 2-19 & $7.4(2.8) ; 2-18$ & 0.01 \\
\hline Physicians, mean (SD); range & 3.6 (1.8); 1-9 & 2.8 (1.5); 0-8 & $<0.001$ \\
\hline Pharmacies, mean (SD); range & $1.3(0.6) ; 1-3$ & $1.3(0.5) ; 1-3$ & 0.42 \\
\hline Has help with medications, $\%$ & 16 & 16 & 1.00 \\
\hline Uses medication aid ${ }^{\mathrm{c}}, \%$ & 70 & 57 & 0.06 \\
\hline Pill box, \% & 47 & 50 & 0.67 \\
\hline Written list of medications, \% & 30 & 16 & 0.02 \\
\hline Other, $\%$ & 16 & 6 & 0.02 \\
\hline Shows written list to physician, \% & 18 & 11 & 0.16 \\
\hline Has some form of prescription drug coverage, \% & 91 & 94 & 0.42 \\
\hline Could not purchase meds due to cost, \% & 12 & 28 & 0.005 \\
\hline $\begin{array}{l}\text { Short-test of functional health literacy in adults (S-TOFHLA); } \\
\text { mean (SD); range }\end{array}$ & 24.2 (10.6); 0-36 & 14.8 (11.9); 0-35 & $<0.001$ \\
\hline Adequate & 58 & 29 & \\
\hline Marginal & 12 & 13 & \\
\hline Inadequate & 30 & 58 & \\
\hline Lawton Instrumental Activities of Daily Living Scale (IADLs); ${ }^{\mathrm{e}}$ mean (SD); range & 13.7 (3.0); $1-16$ & 13.5 (3.0); 4-16 & 0.69 \\
\hline
\end{tabular}

ancludes all medications (i.e., prescription, over-the-counter, and complementary and alternative medications)

${ }^{b}$ Defined as any chronic condition documented in the medical record

${ }^{c}$ Does not add up to $100 \%$, since patients may have been using more than one medication aid

${ }^{d}$ Scores range from 0 to 36 and are categorized as inadequate (0-16), marginal (17-22), or adequate (23-36) health literacy

${ }^{e}$ Scores range from 0 (low function, dependent) to 16 (high function, independent)

$N S=$ not significant $(p>0.05)$

remained even after adjustment for our a priori confounders. When removing nonadherence from the model, the mean number of medication-related problems remained significantly greater for blacks than whites (4.34 versus 3.97; adjusted $\mathrm{p}=0.037$ ). There was also a trend towards blacks having significantly more problems related to monitoring $(0.34$ vs. $0.18, \mathrm{p}=0.05$ and $\mathrm{p}=0.06$, covariate adjusted).

\section{DISCUSSION}

Efforts to measure and improve the quality of medication use in older adults have traditionally focused on specific problems, pre-defined quality indicators, or individual diseases (even when patients have multiple chronic conditions). ${ }^{11,12,24-27}$ While important, each approach fails to consider the patient

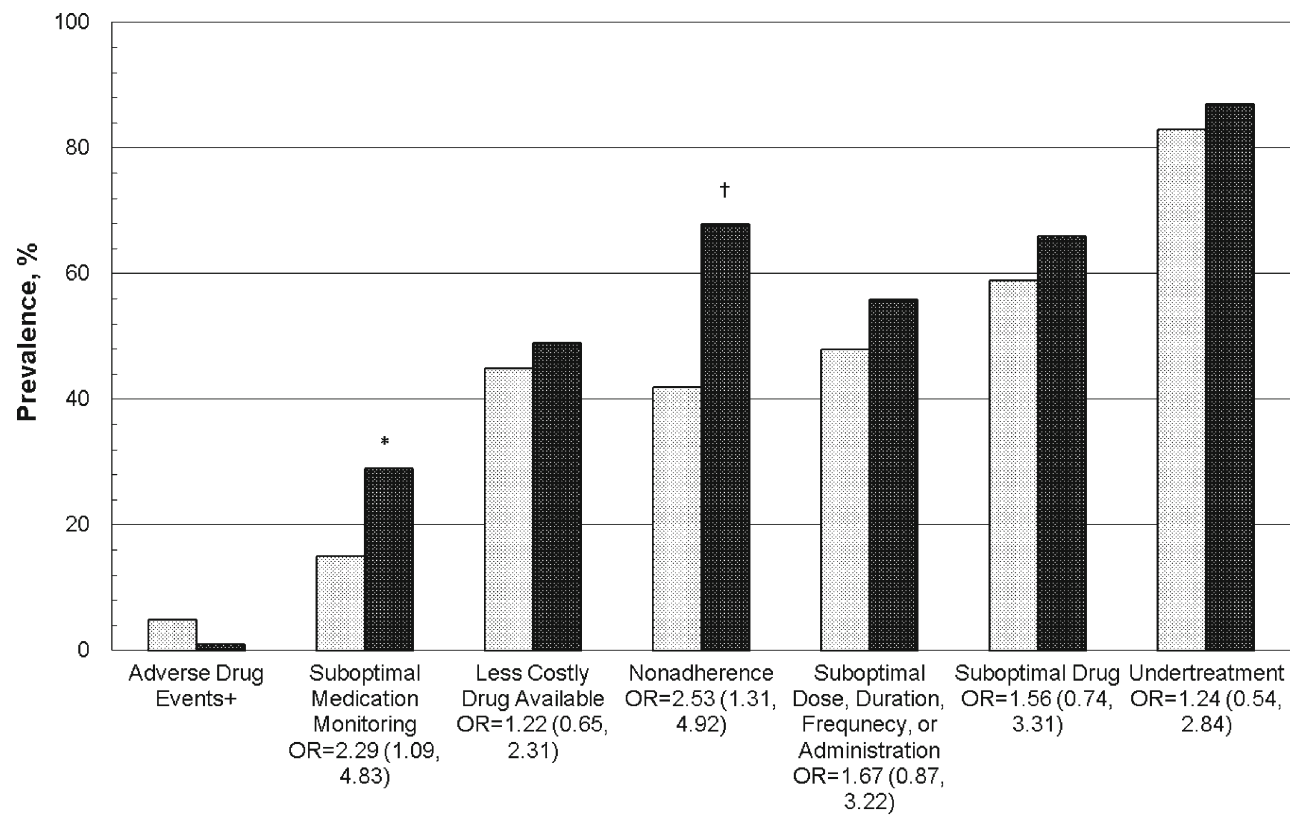

Potential Medication-Related Problems

Figure 1. Prevalence of medication-related problems in community-residing older adults $(n=199)$, with prevalence defined as the number of older adults with any of the drug therapy problems/number of older adults in the sample (unadjusted). Odds ratios (OR) and $95 \%$ confidence intervals for race are included for the covariate adjusted models with White race being the reference category. ${ }^{*} p<0.05,{ }^{\dagger} p<0.01,{ }^{+}$too few events to calculate OR, White race ( $n=99$, white bar), Black race ( $n=100$, shaded bar). 
Table 4. Medication-Related Problems in Community-residing Older Adults $(n=199)$

\begin{tabular}{|c|c|c|c|c|}
\hline & White, $(n=99)^{a}$ & Black, $(n=100)$ & P-value & $\begin{array}{l}\text { Covariate-adjusted }^{e} \\
\text { p-value }\end{array}$ \\
\hline Overall, raw number & 470 & 623 & & \\
\hline Overall, mean (SE) & $4.87(0.26)$ & $6.19(0.30)$ & 0.001 & $<0.001$ \\
\hline Adverse drug events ${ }^{\mathrm{b}, \mathrm{c}}$, mean (SE) & $0.05(0.02)$ & $0.01(0.01)$ & - & - \\
\hline Drug cost, mean (SE) & $0.59(0.08)$ & $0.68(0.08)$ & 0.44 & $0.19^{c}$ \\
\hline Suboptimal medication monitoring, mean (SE) & $0.18(0.04)$ & $0.33(0.06)$ & 0.05 & 0.06 \\
\hline Nonadherence, mean (SE) & $0.89(0.13)$ & $1.84(0.24)$ & $<0.001$ & 0.002 \\
\hline Suboptimal dose, duration, frequency, or administration, mean (SE) & $0.73(0.09)$ & $0.71(0.08)$ & 0.87 & 0.51 \\
\hline Suboptimal drug, mean (SE) & $1.15(0.14)$ & $1.21(0.14)$ & 0.76 & 0.12 \\
\hline Undertreatment $^{\mathrm{c}}$, mean (SE) & $1.22(0.11)$ & $1.33(0.12)$ & $0.48^{\mathrm{d}}$ & $0.92^{\mathrm{d}}$ \\
\hline
\end{tabular}

All means and p-values are adjusted for a fixed pharmacist effect unless otherwise noted

${ }^{a}$ Medication-related problems were not assessed for one individual because medical records were not available for review

${ }^{b}$ Unable to calculate $p$-value due to scarce number of events experienced

${ }^{c}$ Raw means presented

${ }^{d}$ No pharmacist effect included; model would not converge

${ }^{e}$ Adjusted for total number of medications, health literacy, gender, and age

and the multiple, co-existing medication-related problems that may be present. We conducted a longitudinal cohort study to examine the breadth and scope of medication-related problems among community-dwelling older adults and designed the study to examine racial differences in these problems.

Results from the baseline data highlight the prevalence of medication-related problems in community-dwelling older adults. All individuals in our study had at least one clinically important medication-related problem. Moreover, we found significant differences between whites and blacks with respect to total number of medications, ability to afford and purchase medications, and ability to understand and interpret health information. Perhaps most surprisingly, despite blacks having fewer medications (9.7 vs. 11.6, $\mathrm{p}<0.01)$, they had more medication-related problems $(6.2$ versus $4.9, \mathrm{p}<0.01)$. The most common problems for both races were undertreatment, suboptimal drug use, suboptimal dosing, and nonadherence. However, the magnitude of non-adherence was far greater among blacks than whites (1.84 vs. 0.89). Prior work suggests that racial differences exist in medication use and access to care; it is well documented that older black adults have lower total drug spending, use fewer prescription medications, and have higher rates of nonadherence than whites. ${ }^{9,10}$ However, it is unclear what impact race may have on an individual's quality of medication use. Future studies will need to more closely examine factors contributing to racial disparities in medication nonadherence as well as racial disparities in the overall quality of medication use.

Although several studies have been conducted outside the US, only a handful of studies in community-residing older adults in the US have examined the prevalence of multiple, coexisting medication-related problems in individuals. In a study of 183 elderly residents residing independently in federally, subsidized apartment buildings, $75 \%$ had one or more potential medication problems. The most common problems were discrepancies between labeled dosage and dosage actually taken $(37 \%)$, potential drug interactions (27\%), and underuse of needed medication (24\%). ${ }^{28}$ In a study of potential medication-related problems in dually eligible older adults in a Medicaid waiver management program, 49\% were found to have at least one potential medication-related problem, with the most prevalent problem being therapeutic duplication, which occurred in $24 \%$ of individuals. ${ }^{29}$. A large pharmaceu- tical care registry between of patients cared for by pharmacists working in ambulatory care clinics and community pharmacy settings found that $30 \%$ of older adults had at least one drug therapy problem, with the most common problems being additional drug therapy needed (30\%), too low dosage (23\%), and nonadherence (18\%). ${ }^{30}$ A study of 159 older adults in the community pharmacy setting documented 3.9 medicationrelated problems per patient, with the most common problem being undertreatment. This study found that medicationrelated problems increase as patients take more medications and experience more medical problems. ${ }^{31}$

Several factors may account for the higher number of medication-related problems per older adult documented in our study compared to previous studies. ${ }^{30,31}$ First, previous studies used a variety of implicit frameworks for assessing medication-related problems, with some more comprehensively defined and operationalized than others. Second, most previous studies in the community or community pharmacy setting relied on patient interviews and prescription records in formulating their assessments of medication-related problems. In our study, pharmacists conducted thorough medication reviews with each older adult and had access to patients' medical records. Thus, we had more complete information when making an assessment of quality of medication use.

Our study has several limitations. First, patients were recruited from older adults already receiving support from the Department on Aging to maximize their independence. Indeed, our sample was highly functional and independent. Moreover, we excluded individuals with cognitive impairment. Given these factors, the prevalence of medication problems may be even higher among other community-dwelling elders. Second, pharmacists and physicians were not blinded to the race of the patient. However, it would be impossible for either the pharmacist or physician directly responsible for the person's care to be blinded to their patient's race. In addition to those directly involved in the patient's care, future studies could consider utilizing a pharmacist and physician team blinded to race as a final step in the adjudication process. Third, we used an implicit approach to assessing the quality of medication use. This approach allows one to integrate all available information when assessing quality; however, it is less reproducible. Notably, relying solely on explicit measures of quality is not patient-centered and overlooks potentially 
important and significant medication-related problems that often co-exist in older adults. It will be important in future studies to determine the reliability and validity of our measure of quality medication use as well as physician agreement with the pharmacist's assessment of medication-related problems and their acceptance of recommendations to optimize medication therapy. A fourth, and related, point is that, despite training on our medication-related-problem framework, there were differences in the number of problems identified per pharmacist (i.e., on average, one pharmacist documented 1.6 more medication-related problems per patient than the other). Although we adjusted for this difference in our analyses, future studies should seek to enhance the reliability and validity of our measure. Finally, our assessments required travel to patients' homes (medication reviews) and physician offices (medical record reviews). This approach requires significant resources.

\section{CONCLUSION}

Medication-related problems are prevalent in communityresiding older adults and appear to be more prevalent in blacks than whites. Older adults have multiple, co-existing medication-related problems present at a single point in time, with undertreatment, nonadherence, use of a suboptimal drug, and suboptimal dosing being the most prevalent problems in both whites and blacks. Blacks have significantly higher rates of nonadherence than whites. Strategies to more reliably measure the quality of medication use in older adults are needed, and efforts to improve the quality of medication use in older adults must account for potential differences in both the number and types of problems affecting whites and blacks.

These baseline data come from a larger longitudinal study that examines the quality of medication use in white and black older adults over time. Examining the baseline data is an initial and necessary exploratory step toward better defining the elements of quality medication use at the level of the patient. Notably, we have designed our measures to provide the basis for intervening with elderly patients to improve the quality of medication use. It is our hope that such efforts will eventually lead to improvements in the quality of medication use and health outcomes for the older adult population.

Acknowledgment: Dr. Roth had full access to all of the data in the study and takes responsibility for the integrity of the data and the accuracy of the data analysis.

The authors wish to thank Robin H. Zook (RHZ), PharmD, CGP, MTM Pharmacy Associates, for her assistance in subject recruitment, enrollment, and data collection.

The authors wish to thank Ryan Owenby, PharmD, for his assistance in project management and data entry (Ryan was a Doctor of Pharmacy student and a student research assistant, UNC Eshelman School of Pharmacy, University of North Carolina at Chapel Hill, when this study was ongoing).

The authors wish to thank John Paul Timmins, MBA, Planning Systems Productivity \& Co., for designing, developing, and maintaining the study database.

Funding Sources: Drs. Roth and Ivey receive funding from the National Institutes of Health, National Institute on Aging (Principal Investigator (MTR): Research and Career Development Award). The funding agency has no role in the design and conduct of the study, data collection, management, analysis, interpretation of the data, or preparation, review, or approval of this manuscript.

Drs. Esserman and Weinberger receive funding from the National Institutes of Health (CTSA UL1RR025747). The funding agency has no role in the design and conduct of the study, data collection, management, analysis, interpretation of the data, or preparation, review, or approval of this manuscript.

The study was also supported by an American College of Clinical Pharmacy (ACCP) Frontiers Fund Research and Career Development Award.

Conflict of Interest: There are no conflicts of interest or financial interests to declare for any of the four authors.

Corresponding Author: Mary T. Roth, PharmD, MHS; Division of Pharmaceutical Outcomes and Policy, UNC Eshelman School of Pharmacy, University of North Carolina at Chapel Hill, Campus Box 7360, Kerr Hall, Chapel Hill, NC 27599-7360, USA (e-mail: mroth@unc.edu).

\section{REFERENCES}

1. Hohl CM, Dankoff J, Colacone A, Afilalo M. Polypharmacy, adverse drug-related events, and potential adverse drug interactions in elderly patients presenting to an emergency department. Ann Emerg Med. 2001;38:666-71.

2. Higashi T, Shekelle PG, Adams JL, et al. Quality of care is associated with survival in vulnerable older patients. Ann Intern Med. 2005;143:274-81.

3. Landi F, Russo A, Liperoti R, et al. Anticholinergic drugs and physical function among frail elderly population. Clin Pharmacol Ther. 2007:81:235-41.

4. Fu AZ, Jiang JZ, Reeves JH, Fincham JE, Lin GG, Perri M. Potentially inappropriate medication use and healthcare expenditures in the US community-dwelling elderly. Med Care. 2007;45:472-6.

5. Johnson JA, Bootman JL. Drug-related morbidity and mortality and the economic impact of pharmaceutical care. Am J Health Syst Pharm. 1997;54:554-8.

6. Ernst FR, Grizzle AJ. Drug-related morbidity and mortality: Updating the cost-of-illness model. J Am Pharm Assoc. 2001;41:192-9.

7. Johnson JA, Bootman JL. Drug-related morbidity and mortality. A cost-of-illness model. Arch Intern Med. 1995;155:1949-56.

8. Smedley BD, Stith AY, Nelson AR, eds, for the Committee on Understanding and Eliminating Racial and Ethnic Disparities in Health Care, the Board on Health Sciences Policy, and the Institute of Medicine. Unequal Treatment: Confronting Racial and Ethnic Disparities in Health Care. Washington, DC: National Academy Press; 2003.

9. Gaskin DJ, Briesacher BA, Limcangco R, Brigantti BL. Exploring racial and ethnic disparities in prescription drug spending and use among Medicare beneficiaries. Am J Geriatr Pharmacother. 2006;4:96-111.

10. Saha S, Freeman M, Toure J, Tippens KM, Weeks C, Ibrahim S. Racial and ethnic disparities in the VA health care system: a systematic review. J Gen Intern Med. 2008;23:654-71.

11. Fick DM, Cooper JW, Wade WE, Waller JL, Maclean JR, Beers MH. Updating the Beers criteria for potentially inappropriate medication use in older adults: results of a US consensus panel of experts. Arch Intern Med. 2003;163(22):2716-24.

12. Knight EL, Avorn J. Quality indicators for appropriate medication use in vulnerable elders. Ann Intern Med. 2001;135:703-10.

13. Lohr KN, ed, for the Committee to Design a Strategy for Quality Review and Assurance in Medicare, the Division of Health Care Services, and the Institute of Medicine. Medicare: A Strategy for Quality Assurance. Washington, DC: National Academy Press; 1990.

14. Chassin MR. Quality of care. Time to act. JAMA. 1991;266:3472-3.

15. Roth MT, Moore CG, Ivey JL, Esserman DA, Campbell WH, Weinberger M. The quality of medication use in older adults: methods of a longitudinal study. Am J Geriatr Pharmacother. 2008;6:220-33.

16. Callahan CM, Unverzagt FW, Hui SL, Perkins AJ, Hendrie HC. Sixitem screener to identify cognitive impairment among potential subjects for clinical research. Med Care. 2002;40:771-81.

17. Baker DW, Williams MV, Parker RM, Gazmararian JA, Nurss J. Development of a brief test to measure functional health literacy. Patient Educ Couns. 1999;38:33-42.

18. Lawton MP, Brody EM. Assessment of older people: self-maintaining and instrumental activities of daily living. Gerontologist. 1969;9:179-86. 
19. Strand LM, Morley PC, Cipolle RJ, Ramsey R, Lamsam GD. Drugrelated problems: their structure and function. DICP. 1990;24:1093-7.

20. Shrank WH, Polinski JM, Avorn J. Quality indicators for medication use in vulnerable elders. J Am Geriatr Soc. 2007;55(Suppl 2):S373-82.

21. Kerr EA, Smith DM, Hogan MM, et al. Building a better quality measure: are some patients with poor quality actually getting good care? Med Care. 2003;41(10):1173-82.

22. Morisky DE, Green LW, Levine DM. Concurrent and predictive validity of a self-reported measure of medication adherence. Med Care. 1986;24 (1):67-74.

23. Pagona M, Gauvreau K, eds. Principles of Biostatistics. 2nd ed. Pacific Grove, Calif: Duxbury; 2000.

24. Hanlon JT, Weinberger M, Samsa GP, et al. A randomized, controlled trial of a clinical pharmacist intervention to improve inappropriate prescribing in elderly outpatients with polypharmacy. Am J Med. 1996;100(4):428-37.

25. Fillenbaum GG, Hanlon JT. Racial and ethnic disparities in medication use among older adults. Am J Geriatr Pharmacother. 2006 Jun;4(2):93-5.
26. Murray MD, Young J, Hoke S, et al. Pharmacist intervention to improve medication adherence in heart failure: a randomized trial. Ann Intern Med. 2007 May 15;146(10):714-25.

27. Shrank WH, Asch SM, Adams J, et al. The quality of pharmacologic care for adults in the United States. Med Care. 2006;44:936-45.

28. Ostrom JR, Hammarlund ER, Christensen DB, Plein JB, Kethley AJ. Medication usage in an elderly population. Med Care. 1985 Feb;23 (2):157-64.

29. Alkema GE, Wilber KH, Simmons WJ, Enguidanos SM, Frey D. Prevalence of potential medication problems among dually eligible older adults in Medicaid waiver services. Ann Pharmacother. 2007 Dec;41 (12): 1971-8

30. Cipolle RJ, Strand LM, Morley PC, eds. Pharmaceutical Care Practice. New York, NY: McGraw-Hill; 2004

31. Kassam R, Farris KB, Burback L, Volume CI, Cox CE, Cave A Pharmaceutical care research and education project: pharmacists interventions. J Am Pharm Assoc. 2001;41(3):401-10. 